\title{
miR-888 regulates cancer progression by targeting multiple targets in lung adenocarcinoma
}

\author{
JI-XIANG CAO ${ }^{1,2}$ \\ ${ }^{1}$ School of Life Sciences, Peking University, Beijing 100871; ${ }^{2}$ Department of Pathology, \\ Zhongshan Hospital, Xiamen University, Xiamen, Fujian 361004, P.R. China
}

Received October 29, 2018; Accepted March 22, 2019

DOI: $10.3892 /$ or.2019.7118

\begin{abstract}
Aberrantly expressed miRNAs play a crucial role in the progression of lung adenocarcinoma. However, to date, the role of miR-888 in lung adenocarcinoma progression is unclear. In the present study, the biological function of miR-888 and its underlying mechanism in lung adenocarcinoma progression were explored. RT-qPCR was performed to detect the expression of miR-888 in 38 matched lung adenocarcinoma samples respectively. Next, the effects of miR-888 on the proliferation, invasion and migration of lung adenocarcinoma A549 cells were evaluated by a series of gain- and loss-of-function assays. Our results revealed that miR-888 was significantly upregulated in lung adenocarcinoma tissues, and its expression was markedly associated with clinical staging in patients. Moreover, ectopic expression of miR-888 in vitro was revealed to function as a double-edged sword in the progression of lung adenocarcinoma A549 cells by targeting multiple targets. Overexpression of miR-888 promoted the invasion and migration of lung adenocarcinoma A549 cells by targeting E-cadherin and tissue inhibitor of metalloproteinase 2. In addition, ectopic expression of miR-888 inhibited the proliferation of lung adenocarcinoma A549 cells by targeting cell division cycle 7 (CDC7). In addition, the immunohistochemical results and The Cancer Genome Atlas (TCGA) database revealed that CDC7 was significantly upregulated in lung adenocarcinoma tissues, suggesting that miR-888 may function as an oncogene in the progression of lung adenocarcinoma patients, and the
\end{abstract}

Correspondence to: Dr Ji-Xiang Cao, School of Life Sciences, Peking University, Beijing Summer Palace Road 5, Beijing 100871, P.R. China

E-mail: j_xcao@bjmu.edu.cn

Abbreviations: CDC7, cell division cycle 7; miRNA, microRNA; TIMP2, tissue inhibitor of metalloproteinase 2; TCGA, The Cancer Genome Atlas; NSCLC, non-small cell lung carcinoma; IHC, immunohistochemistry; 3'-UTR, 3'-untranslated region; OS, overall survival; WT, wild-type

Key words: miR-888, lung adenocarcinoma, proliferation, invasion and migration, CDC7, E-cadherin, TIMP2
miR-888/CDC7 axis was not the dominant pathway for CDC7 regulation in patients with lung adenocarcinoma. In conclusion, our findings indicated that miR-888 may act as a potential new therapeutic target for patients with lung adenocarcinoma.

\section{Introduction}

Lung cancer is the most frequent malignancy, causing 1.6 million deaths/year worldwide (1). Approximately $85 \%$ of lung cancers consist of non-small cell lung carcinoma (NSCLC). NSCLC is generally categorized into three major histological subtypes: Lung adenocarcinoma, lung squamous cell carcinoma and large cell carcinoma, and lung adenocarcinoma is the most prevalent form of lung cancer (2). Although treatment strategies for NSCLC have markedly increased in recent years, the estimated 5-year overall survival (OS) remains only $16 \%$ (3). Thus, understanding the molecular mechanisms which regulate the progression of lung adenocarcinoma would lead to improved treatment of patients.

MicroRNAs (miRNAs) are a class of small non-coding RNAs that negatively regulate genes at the post-transcriptional level by directly binding to the 3'-untranslated region (UTR) of target mRNAs; resulting in the degradation of mRNA molecules or the inhibition of their translation $(4,5)$. Moreover, miRNAs are involved in the regulation of multiple biological processes involving cancer, such as tumorigenesis and development, cell proliferation, metastasis, invasion and apoptosis $(6,7)$. In recent years, increasing evidence has revealed the relationships between miRNAs and disease progression of NSCLC. For example, miR-30a/c reduction maintained self-renewal and promoted tumorigenesis in NSCLC-initiating cells by targeting oncogene TM4SF1 (8), while Song et al reported that miR-409 inhibited human non-small cell lung cancer progression by directly targeting SPIN1 (9). Although the function of numerous miRNAs has been reported in lung cancer, the molecular regulatory mechanisms of miRNAs and their effects on lung cancer progression are still not well understood.

Recently, miR-888 was revealed to be upregulated in several types of cancer, such as endometrial and colorectal cancer, and the upregulated levels of miR-888 were correlated with the poor outcome in patients with endometrial and colorectal cancer (10-12). In addition, several studies revealed that miR-888 functioned as an oncogene and modulated cancer 
cell proliferation, invasion and migration (13-16). However, the biological role of miR-888 in lung adenocarcinoma is not fully elucidated.

In the present study, the expression, roles, and mechanisms of miR-888 in the progression of lung adenocarcinoma in A549 cells and human lung adenocarcinoma tissues were investigated, and the results revealed that miR-888 may be a potential new therapeutic target in lung adenocarcinoma.

\section{Materials and methods}

Tissue samples for patients with lung adenocarcinoma. A total of 38 pairs of primary lung adenocarcinoma and adjacent non-tumor tissues were obtained from the Affiliated Zhongshan Hospital of Xiamen University (between January 2014 and June 2016). The clinical characteristics of 38 lung adenocarcinoma patients were as follows: Mean age (range): 62.9 years (45-81 years). Sex: Male, 26 cases; female, 12 cases. Clinical staging: Stage I-II, 16 cases; and stage III-IV, 22 cases. All patients provided informed consent, and all specimens were confirmed by a pathologist. The study was approved by the Research Ethics Committee of Xiamen University (Xiamen, China).

Immunohistochemistry (IHC). For IHC, 4- $\mu$ m thick slides were deparaffinized in xylene and rehydrated in a descending graded series of alcohol dilutions. Antigen retrieval was performed in $10 \mathrm{mM}$ citrate buffer ( $\mathrm{pH}$ 6.0) in a microwave oven at maximum power $(800 \mathrm{~W})$ for $3 \mathrm{~min}$, followed by $15 \mathrm{~min}$ at medium power, and cooling to room temperature. After phosphate-buffered saline (PBS) washes, the slides were blocked with $3 \% \mathrm{H}_{2} \mathrm{O}_{2}$ for $10 \mathrm{~min}$ and blocked with goat serum for $30 \mathrm{~min}$ at room temperature. The sections were incubated with a primary mouse anti-human antibody against cell division cycle 7 (CDC7) (dilution 1:100; cat. no. sc-56275; Santa Cruz Biotechnology, Santa Cruz, CA, USA) overnight at $4^{\circ} \mathrm{C}$. The slides were washed thrice with PBS and then incubated with an HRP-conjugated secondary antibody (dilution 1:250; cat. no. ZDR-5307; ZSGB-BIO, Beijing, China) for $30 \mathrm{~min}$ at room temperature. For all slides, the immune reaction was assessed using diaminobenzidine, and the sections were then counterstained with hematoxylin. For the semiquantitative analysis of CDC7 immunoreactivity, the immunohistochemical score (IHS) was used. Briefly, IHS=SI (staining intensity) x PP (percentage of positive cells). The staining intensity (SI) was categorized into 4 groups (0-3), where 0 was negative, 1 was weak, 2 was moderate, and 3 was strong. The PP was estimated and classified on a five-point positive range score as follows: $0, \leq 5 \%$ staining; $1,6-25 \%$ staining; 2, 26-50\% staining; 3, 51-74\% staining; and 4, $\geq 75 \%$ staining. Cases were categorized into two groups: IHS=0, negative and IHS $\geq 1$, positive (17).

Cell culture. The human non-small cell lung cancer (NSCLC) cell line A549 was obtained from the American Type Culture Collection (ATCC; Manassas, VA, USA). Cells were maintained in Dulbecco's modified Eagle's medium (DMEM) with $10 \%$ fetal bovine serum (FBS; HyClone, Thermo Scientific, Inc., Waltham, MA, USA) and grown at $37^{\circ} \mathrm{C}$ in $5 \% \mathrm{CO}_{2}$.
Plasmid generation, small interfering RNA (siRNAs), and transfection. The pcDNA3.1-CDC7 construct was kindly provided by Dr Peter Cherepanov (Imperial College London, London, UK). For the construction of luciferase reporter plasmids, the full length CDC7 3'-UTR, truncated versions of this UTR, or constructs containing 3'-UTR point mutations were amplified by PCR and inserted into the pMiR-reporter plasmid. Primers were as follows: $C D C 7$ full-length 3'-UTR forward, 5'-GGACTAGTCCTAATGGATCTTCATTTAATGTTTAC-3' and reverse, 5'-CCCAAGCTTGGGTAAAAAATATAAAAG GATAACTTTATTG-3'; Fragment A forward, 5'-GGACTA GTCCTAATGGATCTTCATTTAATGTTTAC-3' and reverse, 5'-CCCAAGCTTAACAGAAACTTTGTGGTCAG-3'; Mut A forward, 5'-CTAACAACATGATCTTCTTTCCTTTAA ACCTACCTAAGTA-3' and reverse, 5'-TACTTAGGTAGG TTTAAAGGAAAGAAGATCATGTTGTTAG-3'; Fragment B forward, 5'-GGACTAGTAAGTTTCTGGATGTTTTA-3' and reverse, 5'-CCCAAGCTTGGGTAAAAAATATAA AAGGATAACTTTATTG-3'; Mut B forward, 5'-CCAAAT GCTTTTCTTTTTTCCTTTGTATATTTTTTCACAC-3' and reverse, 5'-GTGTGAAAAAATATACAAAGGAAAAAA GAAAAGCATTTGG-3'. The CDC7, E-cadherin and TIMP2 siRNA target sequences, miR-888 mimics and inhibitors were synthesized by Shanghai GenePharma Co., Ltd., (Shanghai, China), and the sequences were as follows: CDC7, 5'-AAGCAG UCAAAGACUGUGGAU-3'; E-cadherin, 5'-CAGACAAAG ACCAGGACUA-3'; TIMP2-1, 5'-GGAAAGAAGGAAUAU CUCA-3'; TIMP2-2, 5'-GGAAGUGGACUCUGGAAAC-3', both TIMP2-1 and TIMP2-2 targeted TIMP2, and in order to enhance the efficacy of knockdown, TIMP2-1 and TIMP2-2 were mixed together. For siRNA, miR-888 mimic and inhibitor transfection, $50 \mathrm{nM}$ of synthesized sequences were delivered to the cells using Lipofectamine 2000 (Invitrogen; Thermo Fisher Scientific, Inc.) according to the manufacturer's instructions.

RNA isolation and RT-qPCR. Total RNA was isolated from tissues and cell lines using TRIzol (Invitrogen; Thermo Fisher Scientific, Inc.). For mRNA detection, cDNA was prepared using RevertAid ${ }^{\mathrm{TM}}$ First Strand cDNA Synthesis kit (Thermo Fisher Scientific, Inc.) according to the manufacturer's instructions. Gene expression was quantified by using the Power SYBR-Green PCR Master Mix (Applied Biosystems; Thermo Fisher Scientific, Inc.). Relative gene expression was assessed by standard curves and quantified using the $2^{-\Delta \Delta \mathrm{Cq}}$ method (18). The primers were as follows: $C D C 7$ forward, 5'-AGTGCC TAACAGTGGCTGG-3' and reverse, 5'-CACGGTGAACAA TACCAAACTGA-3'; GAPDH forward, 5'-TGTCAGTGG TGGACCTGACCT-3' and reverse, 5'-AGGGGAGATTCA GTGTGGTG-3'; miR-888 forward, 5'-ACACTCCAGCTG GGTACTCAAAAAGCTGTC-3' and reverse, 5'-TGGTGT CGTGGAGTCG-3'; U6 forward, 5'-CCTGCTTCGGCA GCACA-3' and reverse, 5'-TGGAACGCTTCACGAA-3'. A stem-loop RT primer for miR-888, 5'-CTCAACTGGTGT CGTGGAGTCGGCAATTCAGTTGAGTGACTGAC-3' and a specific RT primer for U6, 5'-AAAATATGGAACGCTTCA CGAATTTGC-3' were used. GAPDH or U6 were used for normalization.

Western blotting. Whole-cell extracts were prepared in RIPA lysis buffer (Beyotime Institute of Biotechnology, 
Beijing, China). The protein concentrations were determined using the BCA Protein Assay reagent kit (Thermo Fisher Scientific, Inc.). Thirty micrograms of the sample were separated by $10 \%$ SDS-PAGE and transferred onto nitrocellulose membranes. Then, the membranes were incubated in blocking solution consisting of $5 \% \mathrm{w} / \mathrm{v}$ non-fat milk in TBST at room temperature for $1 \mathrm{~h}$, immunoblotted with primary antibodies at $4^{\circ} \mathrm{C}$ overnight, and subsequently incubated with a secondary antibody (dilution 1:1,000; cat. nos. ZDR-5306 and ZDR-5307; ZSGB-BIO) for $1 \mathrm{~h}$ at room temperature. The protein band was visualized using SuperSignal West Pico Chemiluminescent Substrate (Pierce Biotechnology, Inc.; Thermo Fisher Scientific, Inc.) and the chemiluminescent image system (ChemiDoc Touch; Bio-Rad Laboratories, Hercules, CA, USA) and the densitometry of western blotting was quantified using the ImageJ 1.52a software program (NIH; National Institutes of Health, Bethesda, MD, USA). The antibodies used for western blotting were as follows: CDC7 (dilution 1:1,000; cat. no. sc-56275; Santa Cruz Biotechnology), TIMP2 (dilution 1:1,000; cat. no. CST-5738; Cell Signaling Technology, Danvers, MA, USA), E-cadherin (dilution 1:1,000; cat. no. CST-14472; Cell Signaling Technology) and $\alpha$-tubulin (dilution 1:2,000; cat. no. PM054; MBL, Medical \& Biological Laboratories, Nagoya, Japan).

Luciferase reporter assay. For the luciferase reporter assay, A549 cells were co-transfected with luciferase reporter plasmids and miR-888 mimics in 24-well plates. Cells were harvested $48 \mathrm{~h}$ after transfection. The luciferase reporter assay was performed using the Dual-Luciferase reporter assay system (Promega Corporation, Madison, WI, USA) according to the manufacturer's instructions.

Wound-healing assay. Cell migration was assayed using a wound healing assay. A549 cells were transfected with the indicated molecules, such as miR-888 mimics, miR-888 inhibitors and CDC7 siRNA. Forty-eight hours after transfection, A549 cells were seeded into 6-well plates. A scratch was generated in the cell monolayer using a $10-\mu 1$ pipette tip after A549 cells had grown to a confluence of $80-90 \%$. Cells were gently washed three times with PBS to remove the cellular debris and incubated in serum-free DMEM. Wound areas were captured at the indicated time-points ( 0 and $24 \mathrm{~h}$ ) using light microscopy, and the migration distance was quantified using the ImageJ 1.52a software program $(\mathrm{NIH}$; National Institutes of Health).

Invasion assay. Cell invasion was assayed using 24-well Transwell chambers coated with $250 \mu \mathrm{g} / \mathrm{ml}$ Matrigel. A549 cells were transfected with the indicated molecules, such as miR-888 mimics, miR-888 inhibitors and CDC7 siRNA. Forty-eight hours after transfection, a total of $1 \times 10^{4}$ cells in $200 \mu \mathrm{l}$ serum-free medium were added into the upper chamber, and $500 \mu \mathrm{l}$ DMEM with $10 \%$ FBS was added to the lower well. After $24 \mathrm{~h}$ of incubation, the non-invaded cells on the upper chamber membrane were removed, and the invaded cells on the lower surface of the chamber membrane were fixed with $4 \%$ paraformaldehyde and stained with $0.1 \%$ crystal violet. Cells were counted in five random fields using an inverted microscope. Each assay was repeated at least three times.
Cell proliferation assay. A549 cells were seeded into 96-well plates and treated with the indicated molecules, such as transfected with miR-888 mimics, miR-888 inhibitors, or cotransfected with miR-888 mimics and pcDNA3.1-CDC7. The effect of miR-888 on cell proliferation was measured by CellTiter $96^{\circledR} \mathrm{AQ}_{\text {ueous }}$ Non-Radioactive Cell Proliferation Assay (MTS) (Promega Corporation) according to the manufacturer's instructions.

Colony formation assay. For the colony formation assay, A549 cells after transfection were seeded in 6-well plates $\left(5 \times 10^{2}\right.$ cells/well). After incubation for 10 days, the colonies were washed three times with PBS and fixed with $4 \%$ paraformaldehyde for $15 \mathrm{~min}$, then stained with $0.1 \%$ crystal violet for $15 \mathrm{~min}$. Visible cell colonies (ranging in number from 65 to 362 ) were imaged and counted.

Statistical analysis. All statistical analyses were performed using the software GraphPad Prism 5 (GraphPad Software, Inc., La Jolla, CA, USA). Data are presented as the mean \pm SD. The paired Student's t-test was used to compare the difference in paired lung adenocarcinoma tissue samples and adjacent non-tumor tissues obtained from the same patient, and other statistical analyses were conducted using unpaired Student's t-test for two comparisons. One-way analysis of variance (ANOVA) followed with Bonferroni post hoc test was used for three and more comparisons. All statistical tests were two-tailed. A P-value of $<0.05$ was considered to indicate a statistically significant difference.

\section{Results}

miR-888 is upregulated in the tumors of patients with lung adenocarcinoma. To explore the biological function of miR-888 in lung adenocarcinoma, the expression levels of miR-888 in 38 pairs of lung adenocarcinoma tissues was detected. RT-qPCR revealed that the expression levels of miR-888 in lung adenocarcinoma tissues were significantly higher than in adjacent non-tumor tissues (median $=0.427$ and 0.163 respectively, Fig. $1 \mathrm{~A} ; \mathrm{P}<0.001$ ). In addition, an increase in the level of miR-888 was significantly associated with the clinical stage of patients. The results revealed that patients in advanced stages (stage III and IV, $\mathrm{n}=22$ ) had a much higher miR-888 expression (median $=0.163,0.330$ and 0.498 , respectively) than patients in early stages (stage $\mathrm{I}$ and $\mathrm{II}, \mathrm{n}=16$; $\mathrm{P}<0.001, \mathrm{P}=0.0063, \mathrm{P}<0.001$, respectively; Fig. 1B). These results indicated that miR-888 may play a crucial role in lung adenocarcinoma progression.

Effect of miR-888 on the proliferation of lung adenocarcinoma cells. To investigate the biological function of miR-888 in the progression of lung adenocarcinoma, miR- 888 was overexpressed or silenced in A549 cells transfected with miR-888 mimics and inhibitor. MTS assays were performed to assess the effect of miR-888 on lung adenocarcinoma cell proliferation. The results revealed that overexpression of miR-888 significantly inhibited the proliferation of A549 cells (Fig. 2A; miR-888 mimics vs miR-NC: $\mathrm{P}=0.0117, \mathrm{P}=0.0158, \mathrm{P}=0.0286$; miR-888 inhibitor vs miR-NC: $\mathrm{P}=0.0187, \mathrm{P}=0.0048, \mathrm{P}=0.0165$, respectively), while knockdown of miR-888 in A549 cells 
A

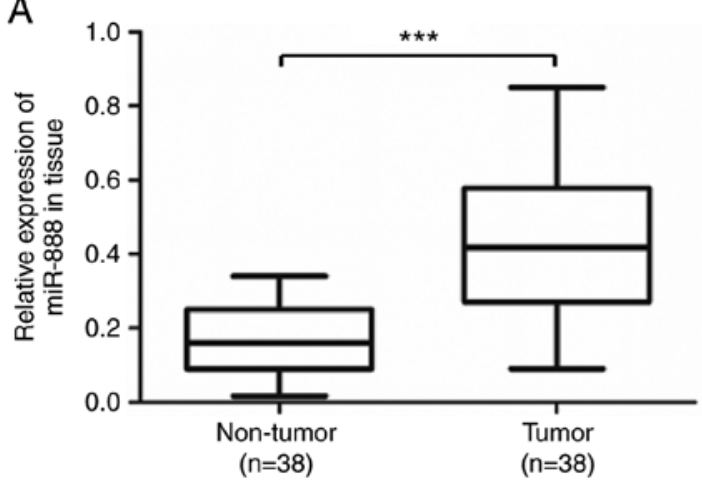

B

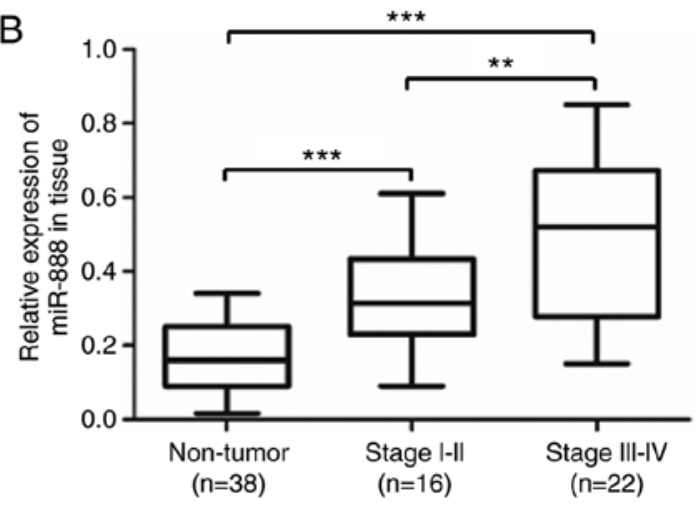

Figure 1. miR-888 is upregulated in lung adenocarcinoma tissues. (A) Expression levels of miR-888 were assessed by RT-qPCR in 38 paired lung adenocarcinoma tissues. (B) The expression levels of miR-888 were significantly higher in patients with advanced stage (stage III and IV, $\mathrm{n}=22$ ) than in patients with early stage (stage I and II, $\mathrm{n}=16$ ). ${ }^{* *} \mathrm{P}<0.01 ;{ }^{* * *} \mathrm{P}<0.001$.

revealed the opposite effect. In addition, colony formation assays also revealed that upregulation of miR-888 inhibited the colony formation capacity of A549 cells and knockdown of miR-888 promoted the colony formation capacity of A549 cells (Fig. 2B; $\mathrm{P}=0.0460, \mathrm{P}=0.0305$, respectively). Collectively, these results indicated that miR-888 suppressed the proliferation of lung adenocarcinoma cells.

Effect of miR-888 on invasion and migration of lung adenocarcinoma cells. To further assess the effect of miR-888 on the invasion and migration potential of A549 cells, Transwell and wound-healing assays were performed. As revealed in Fig. 3A, the Transwell assay revealed that overexpression of miR-888 in A549 cells significantly increased the invasive abilities, while downregulation of miR-888 markedly decreased the invasive abilities (Fig. 3A; $\mathrm{P}=0.0028, \mathrm{P}=0.0081$, respectively). In addition, wound-healing assays revealed that overexpression of miR-888 in A549 cells significantly increased the wound-healing in the miR-888 mimic-transfected A549 cells after $24 \mathrm{~h}$, while downregulation of miR-888 in A549 cells revealed the opposite effect (Fig. 3B, $\mathrm{P}=0.0439, \mathrm{P}=0.0252$, respectively). Collectively, these results demonstrated that overexpression of miR-888 promoted the invasion and migration of lung adenocarcinoma A549 cells.

miR-888 downregulates CDC7 expression by targeting the CDC7 3'-UTR. To further explore the mechanisms underlying miR-888-mediated inhibition of proliferation in lung adenocarcinoma A549 cells, four bioinformatics tools (miRanda, MirTarget2, PITA and RNA hybrid) in the miRecords databases (http://c1.accurascience.com/miRecords/) were used to predict its potential target genes and focused on CDC7 as a potential target gene for miR-888. It has been reported that CDC7 was involved in the proliferation of cancer cells and can act as an oncogene in cancer progression (19-21). To validate whether miR-888 could target CDC7, A549 cells were transfected with miR-888 mimics and miR-888 inhibitors. RT-qPCR was employed to ascertain the transfection efficiency (Fig. 4A and D; P=0.0002, $\mathrm{P}=0.0219$, respectively) and CDC7 expression. RT-qPCR and western blotting revealed that both the CDC7 mRNA and protein levels were significantly reduced following miR-888 overexpression (Fig. 4B and C;
$\mathrm{P}=0.0218)$ whereas they were increased following miR-888 inhibition (Fig. 4E and F; $\mathrm{P}=0.0219$ ).

To further assess whether CDC7 is a target of miR-888, luciferase reporter assays were performed. Wild-type (WT) CDC7 containing a full-length 3'-UTR, the 3'-UTR fragments 'A', 'B' (containing one putative miR-888 target site, respectively) and their mutants (Fig. 4G) were inserted downstream of the pMiR-Reporter vector to generate a series of reporter constructs. Each of these constructs was co-transfected with scrambled siRNA or miR-888 mimics. It was observed that miR-888 mimics significantly decreased luciferase activity in cells transfected with the WT miR- 888 binding site, but not in cells transfected with a construct containing the mutant CDC7 3'-UTR (Fig. 4H; $\mathrm{P}=0.0012, \mathrm{P}=0.0119, \mathrm{P}=0.0279$, respectively). These results indicated that miR-888 downregulates CDC7 expression by directly binding to the CDC7 3'-UTR.

CDC7 is required for miR-888-mediated inhibition of proliferation in lung adenocarcinoma A549 cells. To clarify whether miR-888 inhibited A549 cell proliferation by targeting $\mathrm{CDC} 7$, rescue experiments were performed. miR-888 mimics, and CDC7 overexpression plasmid without the 3'-UTR were co-transfected into A549 cells, and miR-888 inhibitor and small interfering RNAs targeting CDC7 (siCDC7) were co-transfected into A549 cells. MTS assays and western blotting revealed that overexpression of CDC7 abolished the miR-888-mediated inhibition of proliferation in A549 cells (Fig. 5A and C; $\mathrm{P}=0.0260, \mathrm{P}=0.0035$, respectively), while knockdown of $\mathrm{CDC} 7$ markedly attenuated miR-888 inhibitor-mediated promotion of proliferation in A549 cells (Fig. 5B and D; $\mathrm{P}=0.0057, \mathrm{P}=0.0015$, respectively). These results indicated that $\mathrm{CDC} 7$ was required for miR-888-mediated inhibition of proliferation in lung adenocarcinoma A549 cells.

Effect of CDC7 on invasion and migration of lung adenocarcinoma cells. To clarify whether miR-888 promoted A549 cell invasion and migration by targeting CDC7, the effect of CDC7 on cell invasion and migration was first assessed through gain- and loss-of-function experiments. CDC7 was overexpressed or silenced in A549 cells by transfection with pcDNA3.1-CDC7 and CDC7 siRNA. Transwell and 

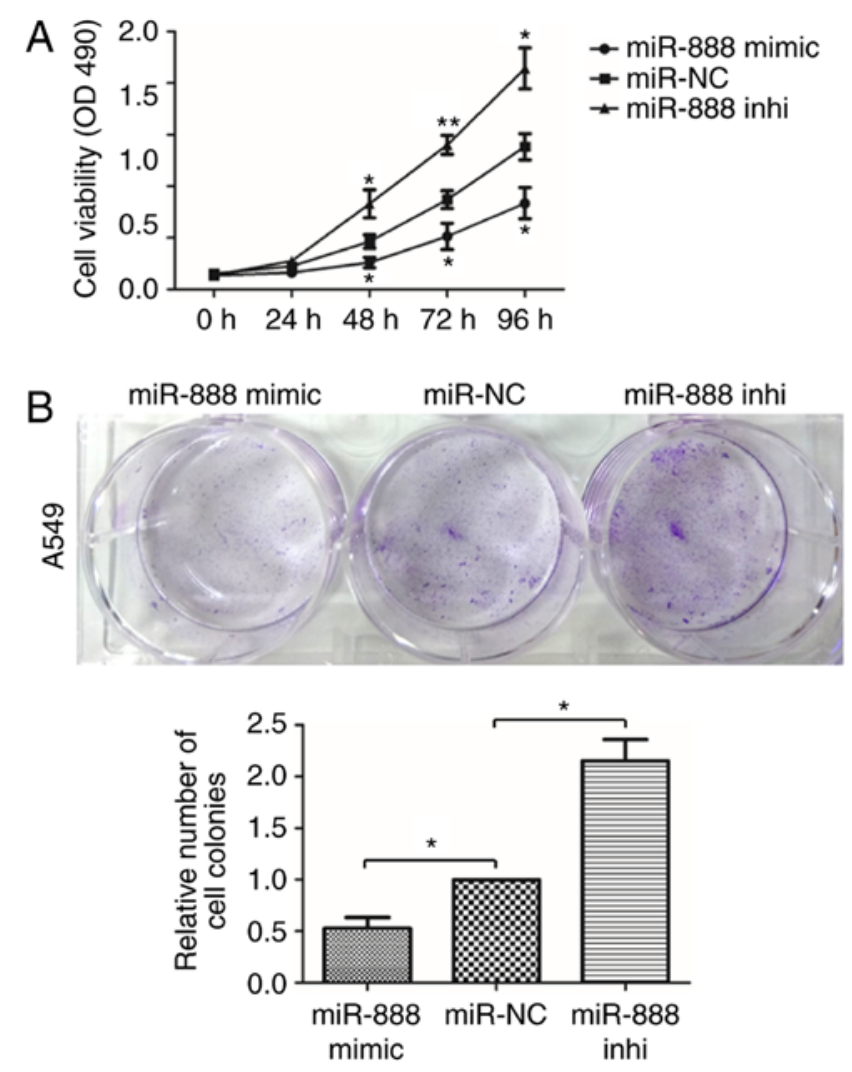

Figure 2. Effect of miR-888 on the proliferation of lung adenocarcinoma A549 cells. (A) The effect of miR-888 on the proliferation in A549 cells was assessed by MTS assays at different time-points. (B) The effect of miR-888 on the proliferation in A549 cells was assessed by colony formation assays. Data are presented as the mean \pm SD. $(\mathrm{n} \geq 3)$. ${ }^{*} \mathrm{P}<0.05 ;{ }^{* *} \mathrm{P}<0.01$.

A
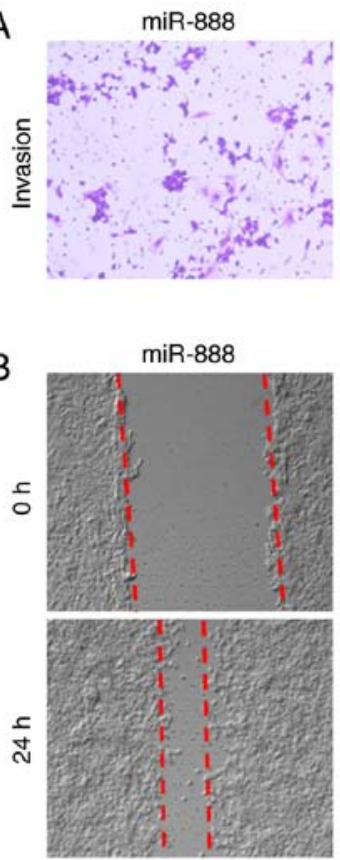

miR-NC

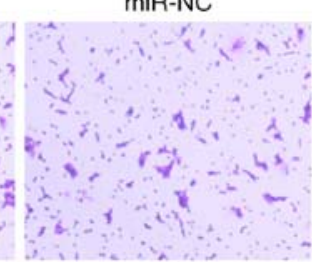

miR-NC
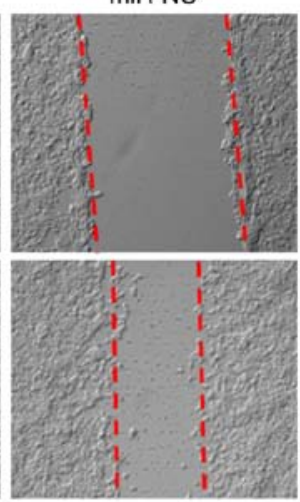

Anti-miR-888

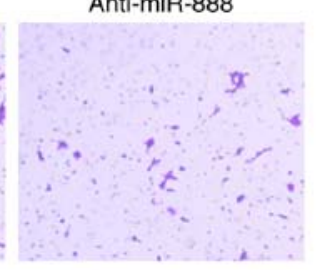

Anti-miR-888
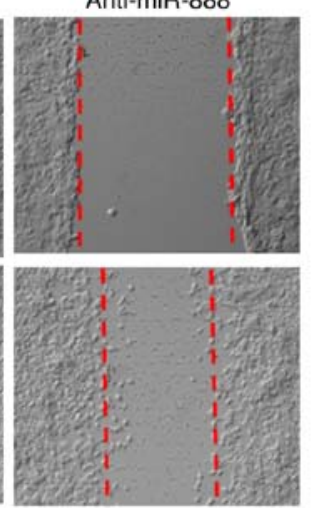
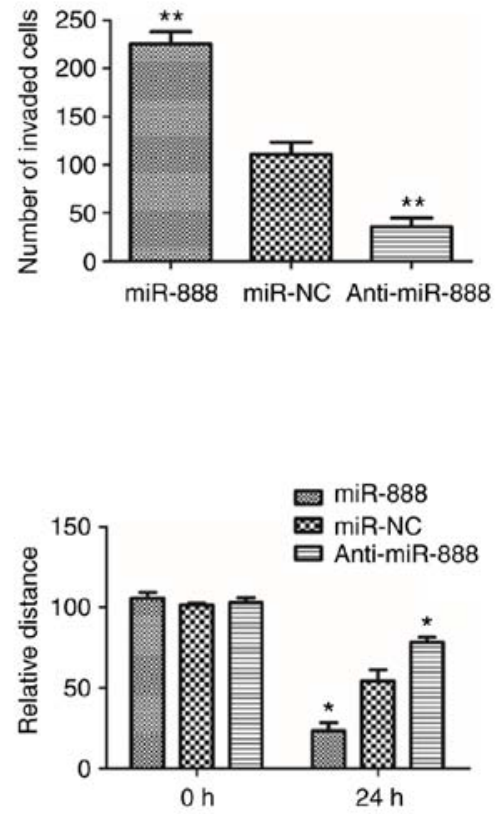

Figure 3. Effects of miR-888 on the invasion and migration of lung adenocarcinoma A549 cells. (A) The effect of miR-888 on the invasion in A549 cells was assessed by Transwell assays. The number of invaded cells were counted and compared in the right diagram. (B) The effect of miR-888 on the migration in A549 cells was assessed by wound-healing assays. The relative distances were calculated and compared in the right diagrams. Data are presented as the mean \pm SD. $(n=3) .{ }^{*} \mathrm{P}<0.05 ;{ }^{* *} \mathrm{P}<0.01$.

wound-healing assays were performed to explore the effects of CDC7 on invasion and migration in A549 cells. The results revealed that overexpression of $\mathrm{CDC} 7$ promoted the invasion and migration of A549 cells (Fig. 6A and B; $\mathrm{P}=0.0319$, 

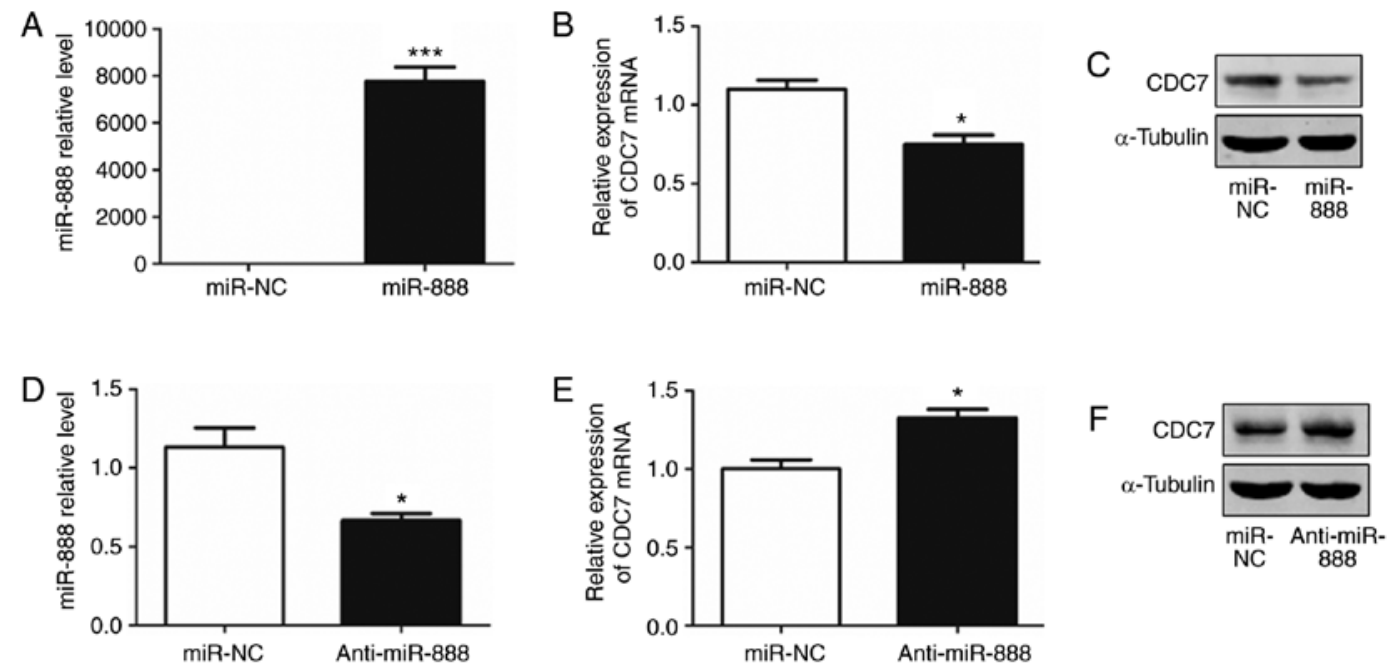

G

$$
\begin{aligned}
& \text { miR-888 } 3^{\prime} \text { ACUGACUGUCGAAAACUCAU } 5^{\prime} \\
& \|\|\|\| \\
& \text { Target A WT } 55^{\prime} \text {...CAACAUGAUCUUCUUUGAGUU.... } 3^{\prime} \\
& \text { MT } 5^{\prime} \ldots \text {...CAACAUGAUCUUCUUU } \underline{C} \underline{C} T U U . . . .3^{\prime} \\
& \text { miR-888 } 3 \text { ' ACUGACUGUCGAAAAACUCAU } 5 \\
& \|\|\|\| \\
& \text { Target BWT } \quad 5^{\prime} \text {...AAUGCUUUUCUUUUUUGAGUU....3' } \\
& \text { MT } \quad 5^{\prime} \ldots \text {...AAUGCUUUUCUUUUUUC_CTUU....3' }
\end{aligned}
$$

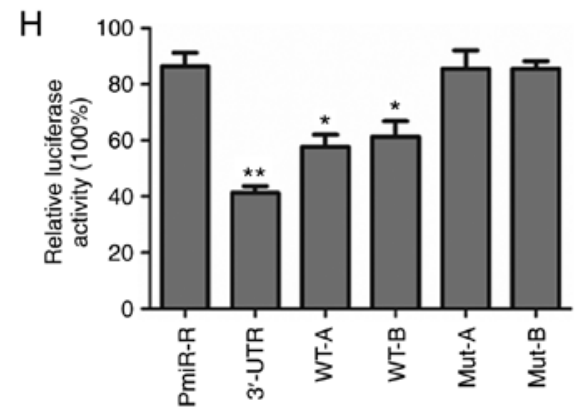

Figure 4. miR-888 downregulates the expression of CDC7 by targeting the CDC7 3'-UTR. Ascertainement of the transfection efficiency of (A) upregulated or (D) downregulated miR-888 expression in A549 cells by RT-qPCR. RT-qPCR analysis of the mRNA level of CDC7 in A549 cells transfected with (B) miR-888 mimics or (E) miR-888 inhibitor. Western blot analysis of CDC7 expression in A549 cells transfected with (C) miR-888 mimics or (F) miR-888 inhibitor. (G) Schematic of the predicted miR-888 binding sequences in the CDC7 3'-UTR and in the mutant 'A' and 'B' CDC7 3'-UTR fragments. (H) Relative luciferase activities of the reporter plasmids in A549 cells. Data are presented as the mean \pm SD. $(n=3) .{ }^{*} \mathrm{P}<0.05 ;{ }^{* * *} \mathrm{P}<0.01 ;{ }^{* * * *} \mathrm{P}<0.001$. CDC7, cell division cycle 7; 3' UTR, 3'-untranslated region.
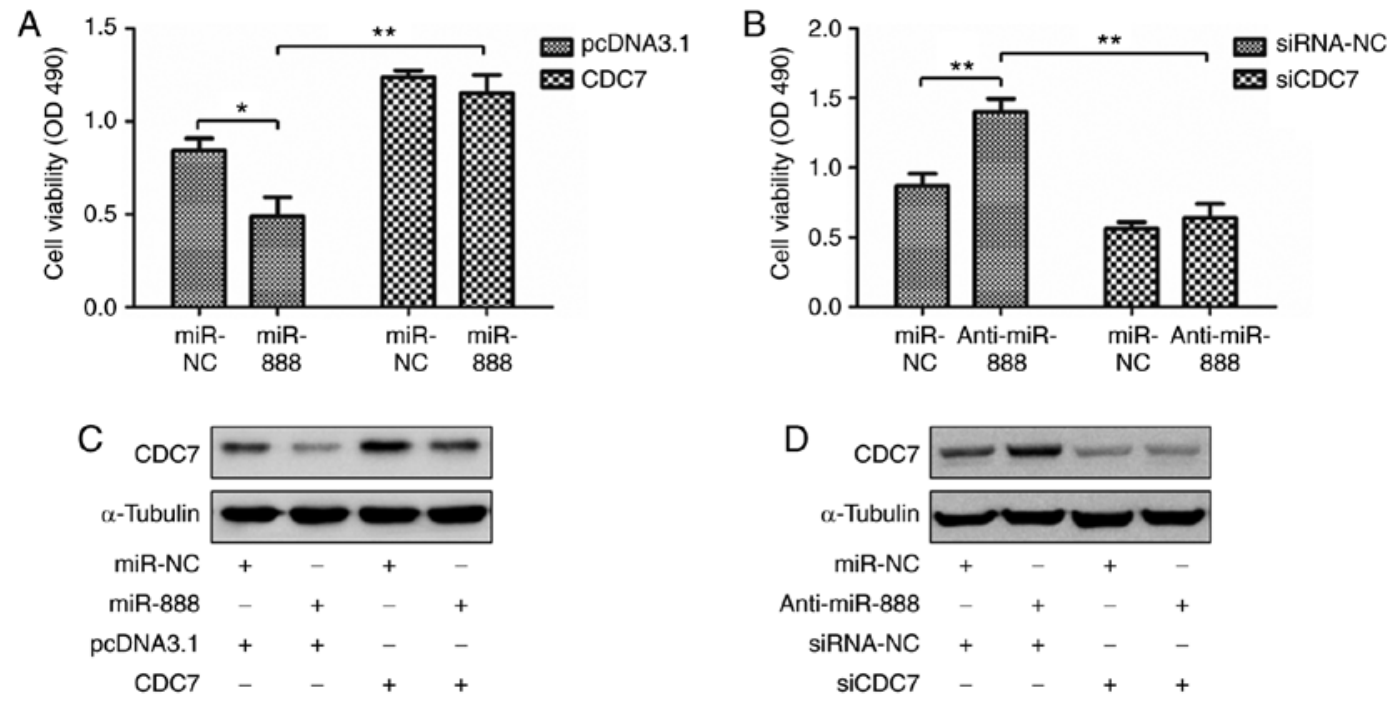

Figure 5. CDC7 is required for miR-888-mediated inhibition of proliferation of lung adenocarcinoma A549 cells. (A and B) A549 cells were co-transfected with (A) miR-888 mimics and CDC7 expression plasmids, and (B) A549 cells were co-transfected with miR-888 inhibitor and CDC7 siRNA. MTS assays were performed to detect cell proliferation in each group. (C and D) The protein expression of CDC7 in A549 cells with different treatments was assessed by western blotting. Data are presented as the mean \pm S00D. $(\mathrm{n}=3) .{ }^{*} \mathrm{P}<0.05 ;{ }^{* *} \mathrm{P}<0.01 . \mathrm{CDC} 7$, cell division cycle 7.

$\mathrm{P}=0.0219$, respectively), and downregulation of $\mathrm{CDC7}$ revealed the opposite effects (Fig. $6 \mathrm{~A}$ and $\mathrm{B} ; \mathrm{P}=0.0463$, $\mathrm{P}=0.0355$, respectively). These results indicated that the
miR-888/CDC7 axis was not the dominant pathway driving the regulation of cell migration and invasion via miR-888 in A549 cells. 

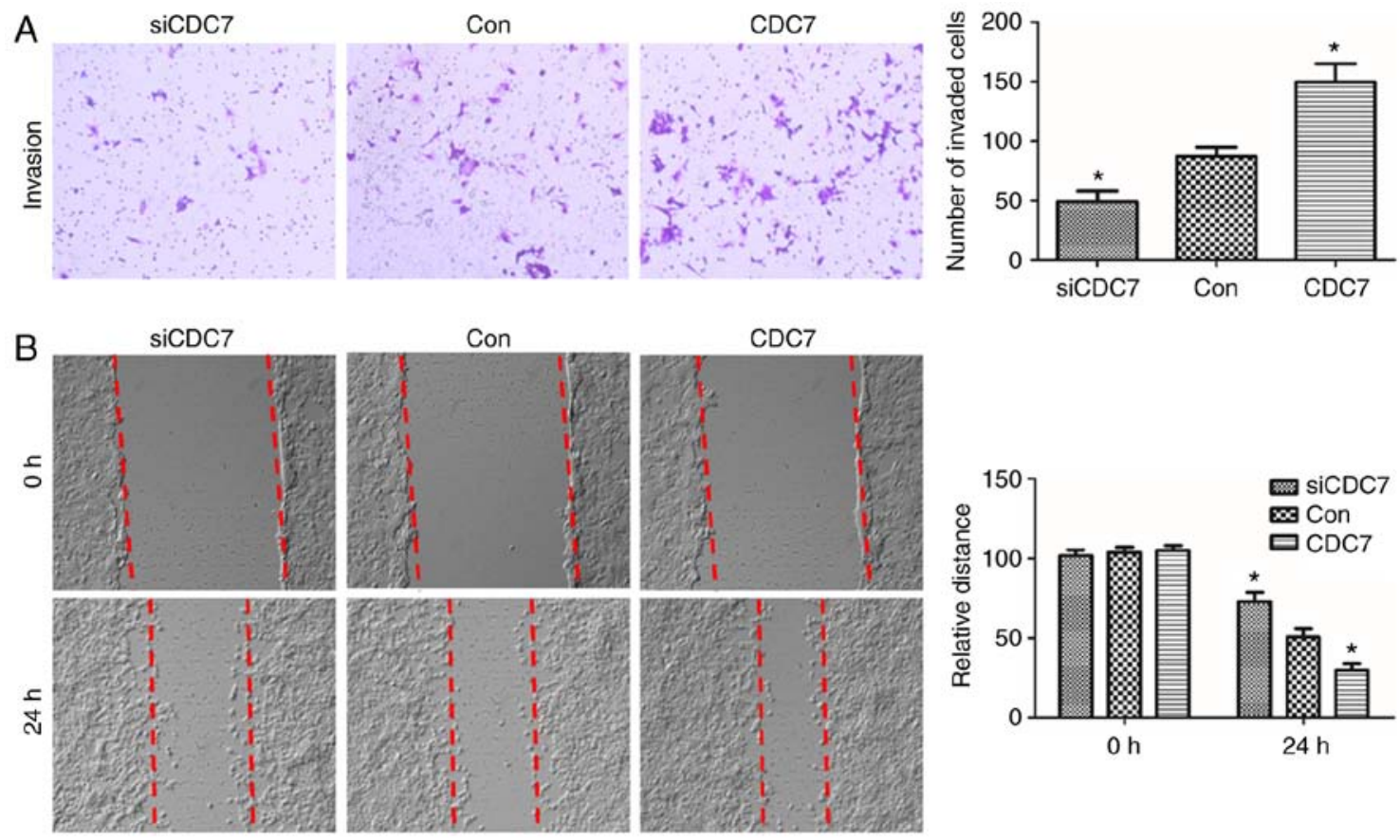

Figure 6. Effects of CDC7 on the invasion and migration of lung adenocarcinoma A549 cells. (A) The effect of CDC7 on the invasion in A549 cells was assessed by Transwell assays. The number of invaded cells were counted and compared in the diagrams. (B) The effect of CDC7 on the migration in A549 cells was assessed by wound-healing assays. The relative distances were calculated and compared in the diagrams. Data are presented as the mean \pm SD. $(n=3)$. ${ }^{*} \mathrm{P}<0.05$. CDC7, cell division cycle 7.
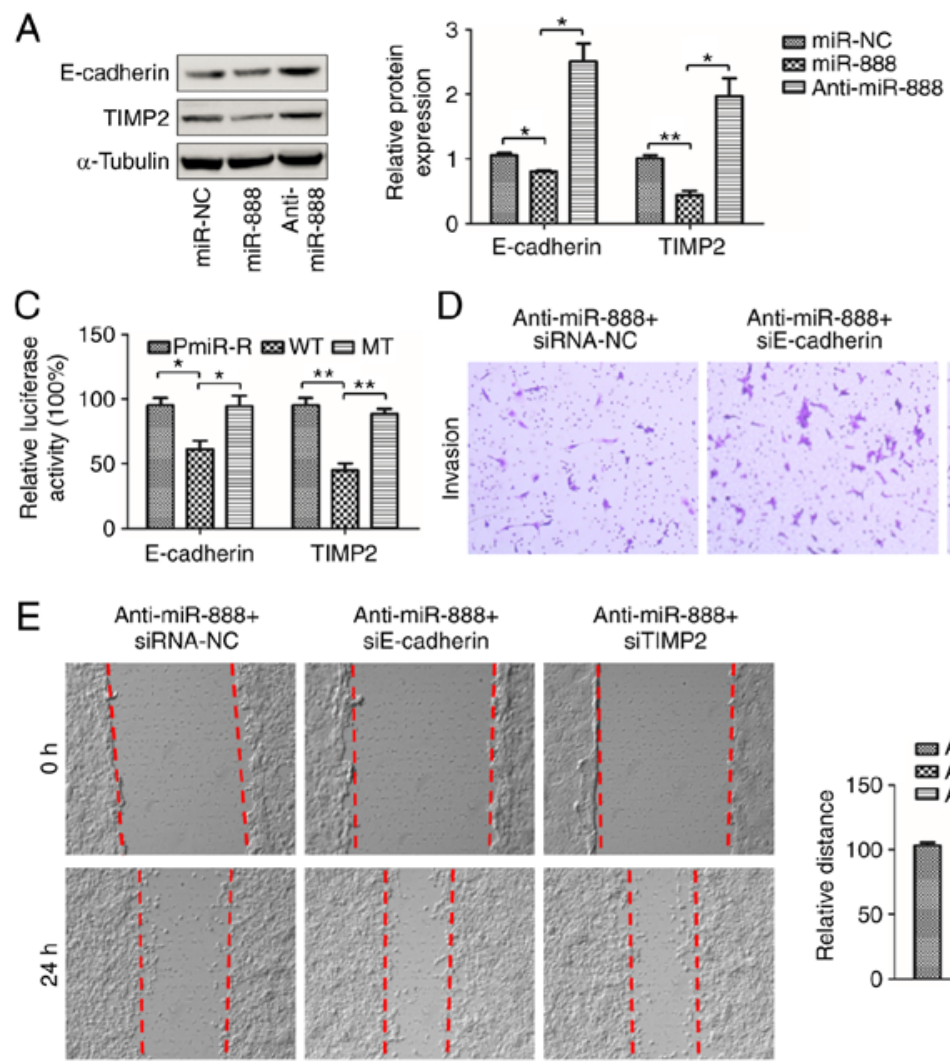

B miR-888 3' ACUGACUGUCGAAAACUCAU 5' \|\|\|\| E-cadherin WT 5'...AUGUCACUGUAGUUUUGAGUG...3

MT $5{ }^{\prime}$...AUGUCACUGUAGUUUUCCCTUG.... $3^{\prime}$ miR-888 $33^{\prime}$ ACUGACUGUCGAAAAACUCAU $5^{\prime}$ 111111 TIMP2-WT 5'...AAAGUAAAGGAUCUUUGAGUA...3

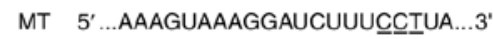
Anti-miR-888 SiTIMP2
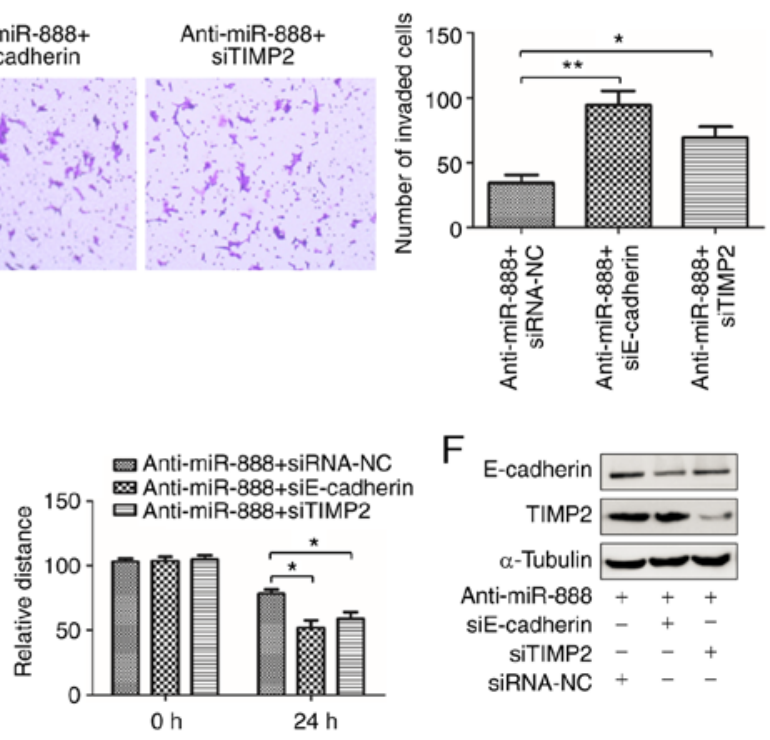

Figure 7. miR-888 promotes invasion and migration of lung adenocarcinoma cells by targeting E-cadherin and TIMP2. (A) A549 cells were transfected with miR-888 mimics or miR-888 inhibitor, and the relative protein levels of E-cadherin and TIMP2 were detected by western blot analysis. (B) Predicted binding sites of miR-888 in wild-type 3'-UTRs of E-cadherin and TIMP2, and mutant 3'-UTRs of E-cadherin and TIMP2. (C) Relative luciferase activities of the reporter plasmids in A549 cells. (D) A549 cells were co-transfected with miR-888 inhibitor and E-cadherin siRNA, and A549 cells were co-transfected with miR-888 inhibitor and TIMP2 siRNA. Transwell assays were performed. The number of invaded cells were counted and compared in the right diagrams. (E) Wound-healing assays were performed. The relative distances were calculated and compared in the right diagrams. (F) The protein expression of E-cadherin and TIMP2 in A549 cells with different treatments was assessed by western blotting. Data are presented as the mean $\pm \mathrm{SD}$. (n=3). ${ }^{*} \mathrm{P}<0.05$; ${ }^{* *} \mathrm{P}<0.01$. TIMP2, tissue inhibitor of metalloproteinase 2; 3'-UTRs, 3'-untranslated regions. 

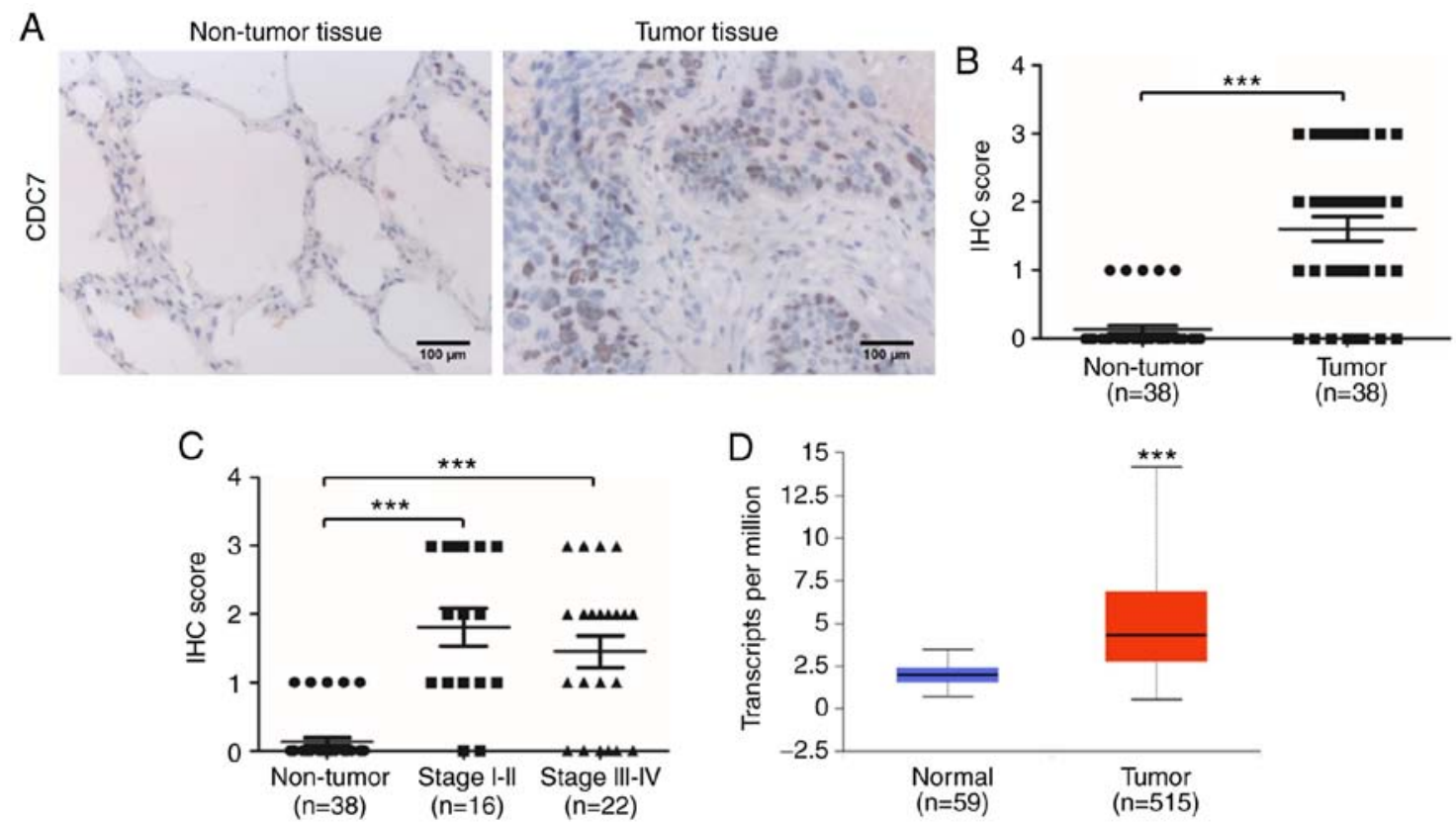

Figure 8. CDC7 is upregulated in lung adenocarcinoma tissues. (A) Representative microphotographs of CDC7 staining in lung adenocarcinoma tissues and adjacent non-tumor tissues, as detected by IHC. (B) The IHC score of CDC7 was determined in lung adenocarcinoma and matched adjacent non-tumor tissues. (C) Expression levels of $\mathrm{CDC7}$ in patients with early stage (stage I and II, $\mathrm{n}=16$ ) and advanced stage (stage III and IV, $\mathrm{n}=22$ ). (D) Boxplot revealing the relative expression of $\mathrm{CDC} 7$ in normal and lung adenocarcinoma tissues from TCGA database. ${ }^{* * * *} \mathrm{P}<0.001$. CDC7, cell division cycle 7; IHC, immunohistochemistry.

miR-888 promotes invasion and migration of lung adenocarcinoma cells by targeting E-cadherin and TIMP2. E-cadherin and TIMP2 have been reported to be targets of miR-888 to promote invasion and migration in breast cancer cells and prostate cancer cells respectively $(15,16)$. Thus, it was speculated that miR-888-induced invasion and migration may be linked to E-cadherin and TIMP2. To demonstrate this hypothesis, a series of experiments were performed. The protein expression of E-cadherin and TIMP2 in A549 cells transfected with miR-888 mimics and inhibitor was first assessed using western blotting assay. The results revealed that overexpression of miR-888 in A549 cells reduced the protein levels of E-cadherin and TIMP2 and knockdown of miR-888 increased the expression of E-cadherin and TIMP2 in A549 cells (Fig. 7A; $\mathrm{P}=0.0402, \mathrm{P}=0.0320, \mathrm{P}=0.0019, \mathrm{P}=0.0182$, respectively). To further assess if miR-888 directly targeted E-cadherin and TIMP2, the putative binding site of miR-888 was predicted and luciferase reporter assays were performed to ascertain the prediction (Fig. 7B). Luciferase reporter assays revealed that miR-888 mimics significantly decreased luciferase activity in cells transfected with the wild-type miR-888 binding site, but not in cells transfected with a construct with the mutant miR-888 binding site, (Fig. $7 \mathrm{C} ; \mathrm{P}=0.0154, \mathrm{P}=0.0309$, $\mathrm{P}=0.0026, \mathrm{P}=0.0024$, respectively). It was next assessed whether miR-888 induced invasion and migration in A549 cells by targeting E-cadherin and TIMP2, by performing rescue experiments. miR-888 inhibitor and small interfering RNAs targeting E-cadherin (siE-cadherin) or TIMP2 (siTIMP2) were co-transfected into A549 cells. Transwell and wound-healing assays revealed that knockdown of E-cadherin markedly attenuated miR-888 inhibitor-mediated suppression of invasion and migration in A549 cells (Fig. 7D and E; $\mathrm{P}=0.0085$, $\mathrm{P}=0.0146$, respectively). Similar results were observed in A549 cells co-transfected with miR-888 inhibitor and TIMP2
siRNA (Fig. 7D and E, right panel; $\mathrm{P}=0.0294, \mathrm{P}=0.0341$, respectively). In addition, western blotting was performed to detect the expression of E-cadherin and TIMP2 in A549 cells co-transfected with miR-888 inhibitor and small interfering RNAs targeting E-cadherin or TIMP2 (Fig. 7F). The data indicated that miR-888 promoted invasion and migration of lung adenocarcinoma cells by targeting E-cadherin and TIMP2.

CDC7 is upregulated in the tumors of patients with lung adenocarcinoma. Our data revealed that ectopic expression of miR-888 in A549 cells exhibited a tumor suppressive function by targeting CDC7, while ectopic expression of miR- 888 in A549 cells exhibited an oncogenic function by targeting E-cadherin and TIMP2. Furthermore, our data revealed that miR-888 was upregulated in the tumors of patients with lung adenocarcinoma and markedly associated with clinical stage progression in patients. Given that $\mathrm{CDC7}$ plays a critical role in 'overexpression of miR-888 as a double-edged sword in the progression of A549 cells', the expression of CDC7 in 38 pairs of lung adenocarcinoma tissues was detected to investigate the role of miR-888 in the tumors of patients with lung adenocarcinoma. IHC results revealed that the protein level of CDC7 was high in lung adenocarcinoma tissues but was low or undetectable in normal lung tissues (Fig. 8A). Furthermore, quantitation data of the IHC score revealed that the protein expression level of $\mathrm{CDC} 7$ was high in lung adenocarcinoma tissues (Fig. 8B; $\mathrm{P}<0.001$ ), however, the expression of $\mathrm{CDC} 7$ was not associated with clinical stage in patients (Fig. 8C). Similar results were observed from UALCAN (http://ualcan. path.uab.edu/), an online database which performs in-depth analyses of TCGA gene expression data (Fig. 8D; P<0.001). Collectively, our data indicated that the miR-888/CDC7 axis did not work in the tumors of patients with lung adenocarcinoma. 


\section{Discussion}

Accumulating evidence has demonstrated that dysregulation of miRNAs (microRNAs) plays essential roles in the development and progression of cancers, such as lung cancer (22). However, the molecular mechanisms of miRNAs that contribute to lung cancer progression have not been fully elucidated. miR- 888 was revealed to be upregulated in several tumors such as colorectal cancer, endometrial cancers and an MCF-7 side population of human breast cancer cells $(10,11,15)$. Consistent with previous studies, the present study study revealed that the expression of miR-888 was significantly upregulated in lung adenocarcinoma samples than in matched non-tumor tissues. Moreover, its expression was asscoiated with the pathologic stage. These findings indicated that miR-888 may function as an oncogene in the progression of lung adenocarcinoma.

It has been reported that the targets of miRNA are vital to the function of miRNA in cells, and one miRNA usually targets multiple genes. Therefore, a miRNA may function as an oncogene or tumor suppressor gene depending on the type of target gene and the gene expression patterns on the tissue and the cell type. For instance, Chen et al reported that miR-766 attenuated cell cycle progression of renal cell carcinoma (RCC) cells by downregulating SF2 expression (23), while Yang et al reported that miR-766 promoted the proliferation and metastasis of hepatocellular carcinoma (HCC) cells by directly decreasing the NR3C2 expression (24). miR-888 has been reported to function as an oncogene in the progression of several cancers. For example, Gao et al reported that miR-888 functioned as an oncogene and predicted poor prognosis in colorectal cancer (11). Huang et al reported that miR-888 promoted cell migration and invasion in breast cancer by targeting E-cadherin (15). Lewis et al reported that miR- 888 promoted cell proliferation in prostate cancer by targeting RBL1 and SMAD4 (13). Hasegawa et al reported that miR-888 promoted cell migration and invasion in prostatic cancer by targeting tissue inhibitor of metalloproteinase 2 (TIMP2) (16). However, the role of miR-888 has never been reported in lung adenocarcinoma. In this study, the results revealed that ectopic expression of miR- 888 functioned as a double-edged sword in the progression of lung adenocarcinoma A549 cells. On the one hand, ectopic expression of miR-888 not only promoted the migration and invasion of lung adenocarcinoma cells by targeting E-cadherin or TIMP2, but also inhibited the migration and invasion of lung adenocarcinoma cells by targeting CDC7. Finally, the function of miR-888 as a promoter in the migration and invasion of lung adenocarcinoma cells suggested that the miR-888/CDC7 axis was not the dominant pathway driving the regulation of cell migration and invasion via miR-888. This result was consistent with the role of miR-888 in colorectal, breast and prostate cancer $(11,14,16)$.

On the other hand, ectopic expression of miR-888 significantly inhibited the proliferation of lung adenocarcinoma cells by targeting $\mathrm{CDC} 7$, while previous studies in colorectal and prostate cancer indicated that miR- 888 promoted cellular proliferation by targeting multiple targets $(11,13)$. These distinct results may indicate that miR-888 regulates cell proliferation depending on the type of cancer cell or the gene expression patterns on the tissue and the cell type. CDC7 is a conserved serine-threonine kinase which plays a crucial role in the initiation of DNA replication $(25,26)$. Thus, miR-888 may exert its inhibitory effect on cell proliferation by blocking CDC7-mediated initiation of DNA synthesis and by inducing G1 arrest. In addition, accumulating data has indicated that CDC7 is overexpressed in many human cancers and tumor cell lines, but has low or undetectable expression in normal tissues and cell lines $(19,27)$ and the overexpression of CDC7 was revealed to be associated with advanced clinical tumor stages, poor survival and chemoresistance $(21,28)$. Therefore, ectopic expression of miR-888 revealed a tumor suppressive function by targeting CDC7 in A549 cells. However, our results revealed that both miR-888 and CDC7 were significantly upregulated in lung adenocarcinoma samples indicating that other potential pathways may involve regulation of CDC7 in lung adenocarcinoma patients, and that the miR-888/CDC7 axis was not the dominant pathway for $\mathrm{CDC} 7$ regulation in patients with lung adenocarcinoma. Furthermore, rescue experiments demonstrated that overexpression of $\mathrm{CDC} 7$ abolished tumor suppressive function of miR-888 in A549 cells (Fig. 5A). Thus, the aforementioned results provided evidence that miR-888 may function as an oncogene in the progression of lung adenocarcinoma patients.

There are several limitations in the present study when interpreting these results. First, our sample size was small, in order to increase the accuracy of the conclusion, increased sample sizes are required. Second, the mechanism underlying the regulation of $\mathrm{CDC7}$ in lung adenocarcinoma patients may be complex and may involve cross-interactions of other signaling pathways, which requires further investigation. Thirdly, further studies are warranted to identify the roles miR-888 in the progression of lung cancer through animal model studies.

In conclusion, our results revealed that ectopic expression of miR-888 in A549 cells functioned as a double-edged sword in the progression of lung adenocarcinoma cells by targeting multiple targets. In addition, ectopic expression of miR-888 significantly inhibited the proliferation of lung adenocarcinoma cells by targeting CDC7; on the other hand, ectopic expression of miR-888 promoted the migration and invasion of lung adenocarcinoma cells by targeting E-cadherin and TIMP2. Furthermore, both miR-888 and CDC7 were upregulated in tumor tissues of patients with lung adenocarcinoma. Thus, our data demonstrated that miR- 888 functioned as an oncogene in the progression of lung adenocarcinoma and may shed light on the therapeutic strategies for lung adenocarcinoma treatment.

\section{Acknowledgements}

Specimens and diagnosis data were provided by the Department of Pathology,Zhongshan Hospital Xiamen University (Xiamen, China). We thank Dr Peter Cherepanov (Imperial College London) for providing the pcDNA3.1-CDC7 constructs.

\section{Funding}

The present study was supported by the National Natural Science Foundation of China (grant no. 81502393). 


\section{Availability of data and materials}

The datasets used during the present study are available from the corresponding author upon reasonable request.

\section{Authors' contributions}

JXC conceived and designed the study, performed the experiments and wrote the manuscript and agrees to be accountable for all aspects of the research in ensuring that the accuracy or integrity of any part of the work are appropriately investigated and resolved.

\section{Ethics approval and consent to participate}

The study was approved by the Ethics Committee of Xiamen University (Xiamen, China), and written informed consents were obtained from the patients.

\section{Patient consent for publication}

Not applicable.

\section{Competing interests}

The authors declare that they have no competing interests.

\section{References}

1. Ferlay J, Soerjomataram I, Dikshit R, Eser S, Mathers C, Rebelo M, Parkin DM, Forman D and Bray F: Cancer incidence and mortality worldwide: Sources, methods and major patterns in GLOBOCAN 2012. Int J Cancer 136: E359-E386, 2015.

2. Siegel RL, Miller KD and Jemal A: Cancer statistics, 2018. CA Cancer J Clin 68: 7-30, 2018.

3. Torre LA, Siegel RL, Ward EM and Jemal A: Global cancer incidence and mortality rates and trends-An update. Cancer Epidemiol Biomarkers Prev 25: 16-27, 2016.

4. Esquela-Kerscher A and Slack FJ: Oncomirs-microRNAs with a role in cancer. Nat Rev Cancer 6: 259-269, 2006.

5. Bartel DP: MicroRNAs: Genomics, biogenesis, mechanism, and function. Cell 116: 281-297, 2004.

6. Hwang HW and Mendell JT: MicroRNAs in cell proliferation, cell death, and tumorigenesis. Br J Cancer 94: 776-780, 2006.

7. Uddin A and Chakraborty S: Role of miRNAs in lung cancer. J Cell Physiol 2018.

8. Ma YS, Yu F, Zhong XM, Lu GX, Cong XL, Xue SB, Xie WT, Hou LK, Pang LJ, Wu W, et al: miR-30 family reduction maintains self-renewal and promotes tumorigenesis in NSCLC-initiating cells by targeting oncogene TM4SF1. Mol Ther 26: 2751-2765, 2018.

9. Song Q, Ji Q, Xiao J, Wang L, Chen Y, Xu Y and Jiao S: miR-409 inhibits human non-small-cell lung cancer progression by directly targeting SPIN1. Mol Ther Nucleic Acids 13: 154-163, 2018.

10. Hovey AM, Devor EJ, Breheny PJ, Mott SL, Dai D, Thiel KW and Leslie KK: miR-888: A novel cancer-testis antigen that targets the progesterone receptor in endometrial cancer. transl Oncol 8: 85-96, 2015.
11. Gao SJ, Chen L, Lu W, Zhang L, Wang L and Zhu HH: miR-888 functions as an oncogene and predicts poor prognosis in colorectal cancer. Oncol Lett 15: 9101-9109, 2018.

12. Bobowicz M, Skrzypski M, Czapiewski P, Marczyk M, Maciejewska A, Jankowski M, Szulgo-Paczkowska A, Zegarski W, Pawłowski R, Polańska J, et al: Prognostic value of 5-microRNA based signature in T2-T3N0 colon cancer. Clin Exp Metastasis 33: 765-773, 2016.

13. Lewis H, Lance R, Troyer D, Beydoun H, Hadley M, Orians J, Benzine T, Madric K, Semmes OJ, Drake R, et al: miR-888 is an expressed prostatic secretions-derived microRNA that promotes prostate cell growth and migration. Cell Cycle 13: 227-239, 2014.

14. Huang $\mathrm{S}$ and Chen L: MiR-888 regulates side population properties and cancer metastasis in breast cancer cells. Biochem Biophys Res Commun 450: 1234-1240, 2014.

15. Huang S, Cai M, Zheng Y, Zhou L, Wang Q and Chen L: miR-888 in MCF-7 side population sphere cells directly targets E-cadherin. J Genet Genomics 41: 35-42, 2014.

16. Hasegawa T, Glavich GJ, Pahuski M, Short A, Semmes OJ, Yang L, Galkin V, Drake R and Esquela-Kerscher A: Characterization and evidence of the miR-888 cluster as a novel cancer network in prostate. Mol Cancer Res 16: 669-681, 2018.

17. Wang L, Liu Q, Lin D and Lai M: CD44v6 down-regulation is an independent prognostic factor for poor outcome of colorectal carcinoma. Int J Clin Exp Patho 8: 14283-14293, 2015.

18. Livak KJ and Schmittgen TD: Analysis of relative gene expression data using real-time quantitative PCR and the $2^{-\Delta \Delta C_{\mathrm{T}}}$ method. Methods 25: 402-408, 2001.

19. Bonte D, Lindvall C, Liu H, Dykema K, Furge K and Weinreich M: Cdc7-Dbf4 kinase overexpression in multiple cancers and tumor cell lines is correlated with p53 inactivation. Neoplasia 10: 920-931, 2008.

20. Li Q, Xie W, Wang N, Li C and Wang M: CDC7-dependent transcriptional regulation of RAD54L is essential for tumorigenicity and radio-resistance of glioblastoma. Transl Oncol 11: 300-306, 2018.

21. Sasi NK, Bhutkar A, Lanning NJ,MacKeigan JP and Weinreich M: DDK promotes tumor chemoresistance and survival via multiple pathways. Neoplasia 19: 439-450, 2017.

22. Del Vescovo V, Grasso M, Barbareschi M and Denti MA: MicroRNAs as lung cancer biomarkers. World J Clin Oncol 5: 604-620, 2014.

23. Chen C, Xue S, Zhang J, Chen W, Gong D, Zheng J, Ma J, Xue W, Chen Y, Zhai W, et al: DNA-methylation-mediated repression of miR-766-3p promotes cell proliferation via targeting SF2 expression in renal cell carcinoma. Int J Cancer 141: 1867-1878, 2017.

24. Yang C, Ma X, Guan G, Liu H, Yang Y, Niu Q, Wu Z, Jiang Y, Bian C, Zang Y and Zhuang L: MicroRNA-766 promotes cancer progression by targeting NR3C2 in hepatocellular carcinoma. FASEB J 33: 1456-1467, 2019.

25. Masai $\mathrm{H}$ and Arai K: Cdc7 kinase complex: A key regulator in the initiation of DNA replication. J Cell Physiol 190: 287-296, 2002.

26. Labib K: How do Cdc7 and cyclin-dependent kinases trigger the initiation of chromosome replication in eukaryotic cells? Genes Dev 24: 1208-1219, 2010.

27. Datta A, Ghatak D, Das S, Banerjee T, Paul A, Butti R, Gorain M, Ghuwalewala S, Roychowdhury A, Alam SK, et al: p53 gain-of-function mutations increase Cdc7-dependent replication initiation. EMBO Rep 18: 2030-2050, 2017.

28. Erbayraktar Z, Alural B, Erbayraktar RS and Erkan EP: Cell division cycle 7-kinase inhibitor PHA-767491 hydrochloride suppresses glioblastoma growth and invasiveness. Cancer Cell Int 16: 88, 2016. 